\title{
Mobility of nonlocal solitons in fading optical lattices
}

\author{
Zhiping Dai.* Xiaohui Ling, Youwen Wang, and Kaiming You \\ Department of Physics and Electronic Information Science, \\ Hengyang Normal University, Hengyang 421008, P.R. China
}

We study the soliton mobility in nonlocal nonlinear media with an imprinted fading optical lattice. The results show that the transverse mobility of solitons varies with the lattice decay rate and the nonlocality degree, which provides an opportunity for all-optical control of light.

PACS numbers: 42.65.Tg, 42.65.Jx, 42.65.Wi 


\section{INTRODUCTION}

In these years, the propagation of solitons in optical lattices has attracted a lot of attention for its unique properties for all-optical control of light [1]. Previous studies include diffraction-managed solitons in curved lattices [2], soliton switching in quasi-continuous optical arrays [3], and soliton control in chirped optical lattices [4]. Longitudinal modulation of lattices offers additional opportunities for soliton control. In particular, periodic longitudinal modulation of the coupling strength results in soliton oscillations and decay [5], harmonic longitudinal modulation of the linear refractive index leads to parametric amplification of transverse soliton swinging [6], while longitudinal modulation of the nonlinearity can be used to steer solitons [7]. Nowadays the technique in fabricating a desired optical lattice is mature. Even the three-dimensional optical lattice has been created with the optical induction technique [8, 9].

Properties of solitons supported by media with local nonlinear response are now well established. However, under appropriate conditions, the nonlinear response of materials can be highly nonlocal, a phenomenon that importantly affects the properties of solitons supported by such media [10]. Some unique properties are discovered in this context. For example, the nonlocality of the nonlinear response can affect the soliton mobility profoundly [11, 12]. Recently, the defect solitons in parity-time symmetric potentials with nonlocal nonlinearity are investigated. It is found that nonlocality can expand the stability region of defect solitons [13].

\section{SOLITON MOBILITY}

In this paper, we study the soliton mobility in nonlocal nonlinear media with an optical lattice that fades away exponentially along the propagation direction. We consider the following dimensionless nonlocal nonlinear Schrödinger equation (NNLSE) [14, 15] with a fading optical lattice [16]

$$
i \frac{\partial \widetilde{U}}{\partial z}+\frac{1}{2} \frac{\partial^{2} \widetilde{U}}{\partial^{2} x}+\widetilde{U} \int R(x-\xi)|\widetilde{U}(\xi, z)|^{2} d \xi+\widetilde{U} p Q(x, z)=0
$$

where $\widetilde{U}(x, z)$ is the complex amplitude envelop of the light beam, $x$ and $z$ are transverse and longitudinal coordinates scaled to the beam width and diffraction length, respectively, $R(x)$ is the real symmetric nonlocal response function, and $p Q(x, z)=p \cos ^{2}(\Omega x) \exp (-\delta z)$ describes the profile of the optical lattice ( $p$ is the lattice depth, $\Omega$ is the lattice frequency, and $\delta$ is the decay rate) (Fig. 1). In practice, such a kind of lattice can be induced optically. Specifically, the refractive index modulation in the transverse direction can be induced with two interfering plane waves [17, 18]. Longitudinal modulation can be created by varying intensities [19], intersection angles, or carrying wavelength [20] of the lattice-forming plane waves.

As previously indicated in Ref. [15], in the strongly nonlocal case if $R(x)$ is twice differentiable at $x=0$, Eq. (1) can be reduced to the following equation

$$
i \frac{\partial \tilde{U}}{\partial z}+\frac{1}{2} \frac{\partial^{2} \tilde{U}}{\partial^{2} x}+\tilde{U} \int\left[R_{0}+\frac{1}{2} R_{0}^{\prime \prime}(x-\xi)^{2}\right]|\tilde{U}(\xi, z)|^{2} d \xi+\tilde{U} p \cos ^{2}(\Omega x) \exp (-\delta z)=0
$$

where $R_{0}=R(0)$ and $R_{0}^{\prime \prime}=R^{\prime \prime}(0)$. For the NNLSE, the beam power is an invariant [21]

$$
P=\int|\tilde{U}|^{2} d x
$$

If we define the beam center as [22]

$$
q(z)=\frac{\int \xi|\tilde{U}(\xi, z)|^{2} d \xi}{\int|\tilde{U}(\xi, z)|^{2} d \xi}
$$

by making use of Eqs. (3) and (4), Eq. (2) is reduced to

$$
\begin{array}{r}
i \frac{\partial \tilde{U}}{\partial z}+\frac{1}{2} \frac{\partial^{2} \tilde{U}}{\partial^{2} x}+\tilde{U} R_{0} P+\frac{1}{2} \tilde{U} R_{0}^{\prime \prime} P(x-q)^{2}+\frac{1}{2} \tilde{U} R_{0}^{\prime \prime} \int(\xi-q)^{2} d \xi \\
+\tilde{U} p \cos ^{2}(\Omega x) \exp (-\delta z)=0 .
\end{array}
$$

By the transformation [22]

$$
U=\widetilde{U} \exp \left\{-i\left[R_{0} P+\frac{R_{0}^{\prime \prime}}{2} \int_{0}^{z} d z^{\prime} \int(\xi-q)^{2}|\tilde{U}(\xi, z)|^{2} d \xi\right] z\right\}
$$




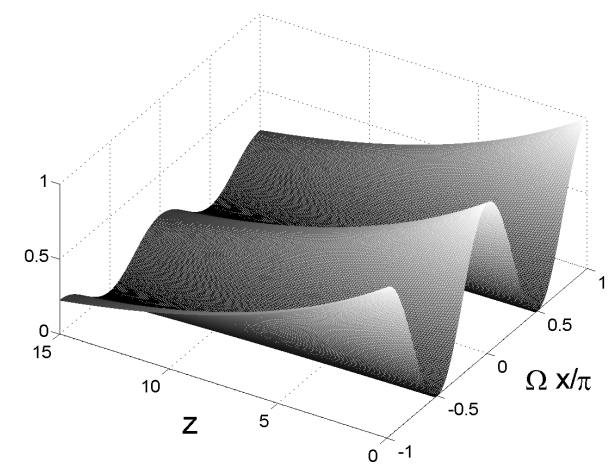

FIG. 1. Profile of a fading optical lattice with $\delta=0.1$ and $p=1$.

Eq. (5) turns into

$$
i \frac{\partial U}{\partial z}+\frac{1}{2} \frac{\partial^{2} U}{\partial x^{2}}-\frac{U}{2} \gamma P(x-q)^{2}+U p \cos ^{2}(\Omega x) \exp (-\delta z)=0,
$$

where $\gamma=-R_{0}^{\prime \prime}>0$.

Comparing Eq. (7) with the one-dimensional Schrödinger equation [23]

$$
i \hbar \frac{\partial}{\partial t} \psi(x, t)=\left[-\frac{\hbar^{2}}{2 m} \frac{\partial^{2}}{\partial x^{2}}+V(x)\right] \psi(x, t),
$$

in an equivalent quantum system with $\hbar=1$ and $m=1$, the equivalent potential energy of Eq. (7) is

$$
V(x)=\frac{1}{2} \gamma P(x-q)^{2}-p \cos ^{2}(\Omega x) \exp (-\delta z) .
$$

With the aid of Ehrenfest theorem [23] and Eqs. (7)- (9), we get an equation of motion for the beam center as follows

$$
\frac{d^{2} q}{d z^{2}}=\frac{\int[-\gamma P(x-q)-\Omega p \exp (-\delta z) \sin (2 \Omega x)]|U|^{2} d x}{\int|U|^{2} d x} .
$$

In the context of strongly nonlocal cases, we employ the following Gaussian-type trial solution

$$
U(x, z)=A \exp \left[-\frac{(x-q)^{2}}{2 w^{2}}\right] \exp [i \beta(x-q)+i \phi],
$$

where $A$ and $\phi$ are the amplitude and phase of the beam respectively, $w$ is the beam width, and $\beta$ is the incident angle with respect to $z$-axis. This choice of ansatz is justified since, in the limit $p \rightarrow 0$, Eq. (11) describes solitons in uniform strongly nonlocal nonlinear media [15]. Substitution of Eq. (11) into Eq. (10) yields

$$
\frac{d^{2} q}{d z^{2}}+\frac{\Omega_{0}^{2}}{2 \Omega} \sin (2 \Omega q)=0
$$

where $\Omega_{0}=\left[2 p \Omega^{2} \exp (-\delta z) \exp \left(-\Omega^{2} w^{2}\right)\right]^{1 / 2}$ defines the frequency of small-amplitude oscillations of the soliton.

Without loss of generality, we assume that a soliton is launched into the medium at the point $x=0$ with an angle $\beta_{0}$, so that $\left.q\right|_{z=0}=0$ and $d q /\left.d z\right|_{z=0}=\beta_{0}$. For $\delta=0$, it has been shown that the soliton's movement is separated by a critical angle $\beta_{c}=\left[2 p \exp \left(-\Omega^{2} w^{2}\right)\right]^{1 / 2}$. When the incident angle is smaller than the critical angle, the soliton is trapped within the waveguide induced by the optical lattices. Above the critical angle, the soliton leaves the waveguide and travels across the lattices [12].

In the presence of a longitudinal modulation, i.e., $\delta \neq 0$, the critical angle $\beta_{c} \sim \Omega_{0} / \Omega=\left[2 p \exp (-\delta z) \exp \left(-\Omega^{2} w^{2}\right)\right]^{1 / 2}$, indicating that $\beta_{c}$ decreases with the propagation distance. As a result, even if the soliton is launched into the lattice with an incident angle $\beta_{0}<\beta_{c}$, it will escape from the central waveguide once the condition $\beta_{0}>\beta_{c}$ is satisfied. In the limit $\delta \rightarrow 0$, the soliton is trapped by the first waveguide and never escapes from the central waveguide. On the contrary, it propagates along its original direction traveling across the lattices without oscillations. As the critical angle depends on the lattice decay rate, a modulation of the decay rate leads to a change of the soliton transverse mobility, a property that might be used for soliton steering. 

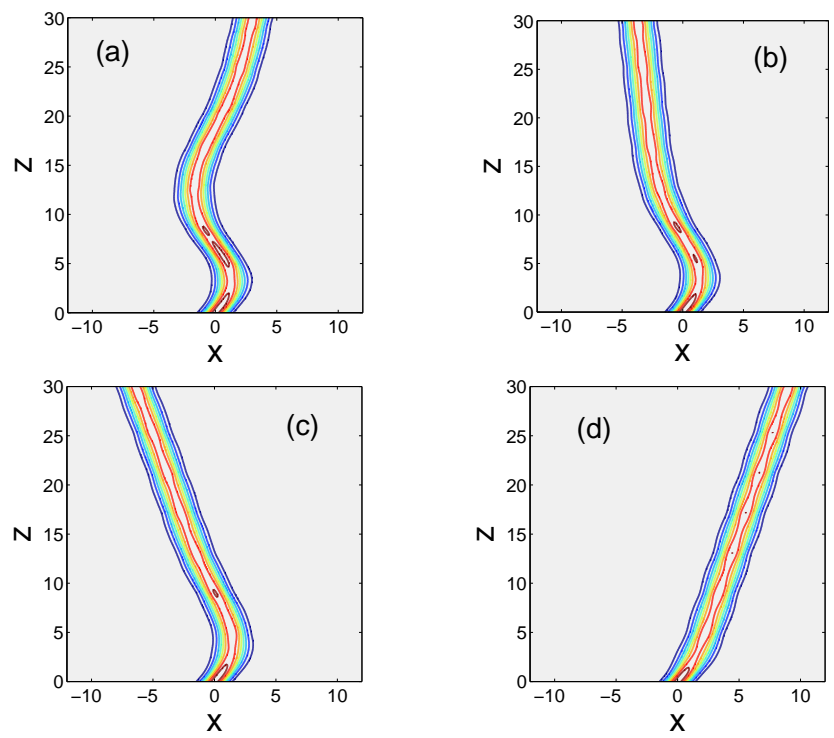

FIG. 2. Contour plots showing the dependence of the soliton mobility on the lattice decay rate. (a) $\delta=0.1$; (b) $\delta=0.15$; (c) $\delta=0.2 ;$ (d) $\delta=0.5$. For all the cases, $\alpha=0.1, p=1, \Omega=0.5$, and $\beta_{0}=0.7$.
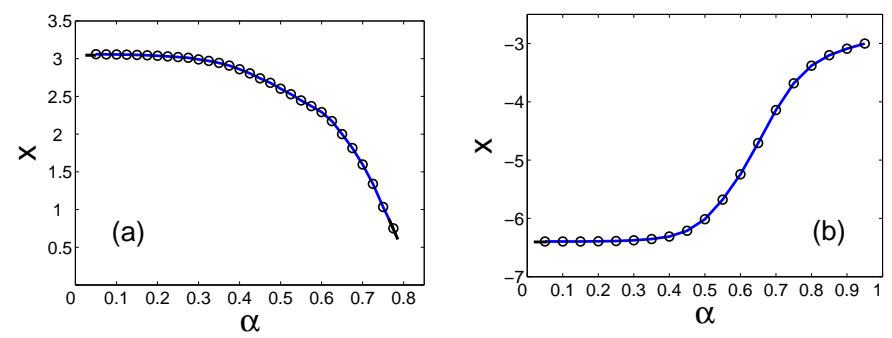

FIG. 3. Dependence of the output position of solitons on the nonlocality degree when the propagation distance is 30 . (a) $\delta=0.1$; (b) $\delta=0.2$. For both cases, $p=1, \Omega=0.5$, and $\beta_{0}=0.7$.

\section{NUMERICAL SIMULATION}

To intuitively show the effect of the lattice and the nonlocality, we numerically simulate the propagation of a Gaussian-type beam in nonlocal nonlinear media with a fading optical lattice. We take Eq. (11) as the evolution equation and $\left.U(x, z)\right|_{z=0}$ in Eq. (11) as the initial condition. The numerical arithmetic we use here is the splitstep Fourier Method [24]. The nonlocal response function is supposed to be a Gaussian function [15], i.e., $R(x)=$ $\exp \left(-x^{2} / 2 w_{m}^{2}\right) /\left(\sqrt{2 \pi} w_{m}\right)$, where we introduce a nonlocal parameter $\alpha$ to define the degree of nonlocality $(\alpha$ is the ratio of the beam width $w$ to the characteristic nonlocal response length $w_{m}$ of the medium). The smaller the nonlocal parameter, the stronger the nonlocality degree.

The dependence of the soliton mobility on the lattice decay rate when $\alpha=0.1$ is shown in Fig. 2, which indicates that the soliton is emitted at different transverse positions with the change of the lattice decay rate. Since the critical angle decreases more quickly for a larger decay rate, an increase of the rate leads to a decrease of the number of soliton oscillations, just as predicted in Sec. 2. Depending on the lattice decay rate, solitons with the angle $\beta_{0}<\beta_{c}$ perform different numbers of oscillations and then start walking freely in diverse directions. When the rate increases to 0.5 , the soliton does not oscillate but is always deflected in the same direction (Fig. 2(d)).

Fig. 3 shows the dependence of the output position of solitons on the nonlocality degree. The curves in Fig. 3 indicate that the nonlocality produces an important effect on the soliton transverse mobility. The reason is that the Peierls-Nabarro potential barrier for solitons moving across the lattice is drastically reduced in the presence of nonlocality, which results in an obvious reduction of the radiation losses of solitons traveling across the lattices [11]. Since the nonlocality makes the radiative trapping less likely to happen, an increase of the nonlocality degree leads to an enhancement of the soliton mobility, as shown in Fig. 3. 


\section{CONCLUSIONS}

In conclusion, we have studied the mobility of solitons in nonlocal nonlinear media with a fading optical lattice. Based on the Ehrenfest theorem, we obtain an expression of the critical angle for the soliton's movement in the strongly nonlocal case, which decreases with the propagation distance. As a result, the soliton always escapes from the central waveguide as long as the critical angle is smaller than the incident angle. It is found through numerical simulations that both the lattice decay rate and the nonlocality degree have important effects on the soliton mobility.

\section{ACKNOWLEDGEMENT}

The work is supported by the Doctorial Start-up Fund of Hengyang Normal University, China (Grant No. 11B42), the Natural Science Foundation of Hunan Province, China (Grant No. 12JJ6001), and the construct program of the key discipline in hunan province, China.

[1] Kartashov Y V, Vysloukh V A and Torner L 2009 Prog. Opt. 5263

[2] Szameit A et al 2008 Phys. Rev. A 78031801

[3] Kartashov Y V, Zelenina A S, Torner L and Vysloukh V A 2004 Opt. Lett. 29766

[4] Kartashov Y V, Vysloukh V A and Torner L 2005 J. Opt. Soc. Am. B 221356

[5] Pertsch T, Peschel U and Lederer F 2003 Chaos 13744

[6] Kartashov Y V, Torner L and Vysloukh V A 2004 Opt. Lett. 291102

[7] Assanto G et al 2010 Phys. Rev. Lett. 104053903

[8] Zhang P, Egger R and Chen Z 2009 Opt. Express 1713151

[9] Xavier J, Rose P, Terhalle B, Joseph J and Denz C 2009 Opt. Lett. 342625

[10] Królikowski W et al 2004 J. Opt. B: Quantum Semiclass Opt. 6 S288

[11] Xu Z Y, Kartashov Y V and Torner L 2005 Phys. Rev. Lett. 95113901

[12] Dai Z P, Wang Y Q and Guo Q 2008 Phys. Rev. A 77063834

[13] $\mathrm{Hu} \mathrm{S}$ M et al 2012 Phys. Rev. A 85043826

[14] Bang O, Królikowski W, Wyller J and Rasmussen J J 2002 Phys. Rev. E 66046619

[15] Guo Q, Luo B, Yi F, Chi S and Xie Y 2004 Phys. Rev. E 69016602

[16] Dai Z P, Lu S Z and You K M 2013 Chin. Phys. B 22014211

[17] Fleischer J W, Carmon T, Segev M, Efremidis N K and Christodoulides D N 2003 Phys. Rev. Lett. 90 023902

[18] Yang R and Wu X 2008 Opt. Express 1617759

[19] Zhang P, Efremidis N K, Miller A, Hu Y and Chen Z 2010 Opt. Lett. 353252

[20] Bergstrom R W, Russell P B and Hignett P 2002 J. Atmos. Sci. 59567

[21] Yakimenko A I, Lashkin V M and Prikhodko O O 2006 Phys. Rev. E 73, 066605

[22] Ouyang S G, Hu W and Guo Q 2007 Phys. Rev. A 76, 053832

[23] Greiner W 2001 Quantum Mechanics: An Introduction, 4th ed. ( Springer-Verlag, Berlin).

[24] Agrawal G P 2007 Nonlinear Fiber Optics (San Diego: Academic) 